\title{
What are the Predictors of Coronary Artery Disease in Young Saudi Arabian Patients
}

\begin{abstract}
Background: Coronary artery disease (CAD) is defined as obstruction of the lumen of the coronary artery due to formation of atherosclerotic plaque. This eventually leads to reduced blood supply to the heart. It could lead to high morbidity and mortality. Risk factors for CAD are divided into modifiable or nonmodifiable. Modifiable such as diabetes, hypertension, hyperlipidemia, obesity, smoking while non-modifiable risks are family history, age, and gender according to previous studies.
\end{abstract}

Objectives: The aim of this study is to identify the predictors and the prevalence of coronary disease risk factors among young Saudi patients.

Methods: This study reviewed 1061 patients referred to the cardiac catheterization lab in King Abdulaziz Cardiac Center in Riyadh between the year 2008 and 2013. Included patients were males and females aged 18-45 years who underwent coronary angiography for various clinical indications.

Results: The results were divided into two groups; Group A aged $<35$ and Group B >35years. Male gender in Gp.A 143(16.1\%) vs. Gp.B 746(83.9\%) p-value= 0.500. Smoking Gp.A $84(15.8 \%)$ vs. Gp.B $446(84.2 \%)$ p=0.680. Obesity Gp.A $43(14.1 \%)$ vs. Gp.B 262(85.9\%) p=0.234. Family history Gp.A $24(23.1 \%)$ vs. $80(76.9 \%)$ p=0.069. Hyperlipidemia Gp.A $40(12.9 \%)$ vs. Gp.B 271(87.1\%) $\mathrm{p}=0.045$. Hypertension Gp.A 20(8.2\%) vs. Gp 233(91.8\%) $\mathrm{p}=<0.001$. Diabetes Gp.A 20(7.2\%) vs.Gp.B $257(92.8 \%) \mathrm{p}=<0.001$

Conclusion: The prevalence of CAD was found the highest in male gender, smoking, and obesity respectively. In patients $>35$ years we found that HTN and Diabetes were statistically significant risk factors. Therefore, a modification of lifestyle habits is recommended.

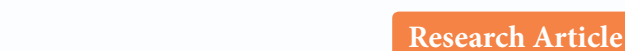

Volume 4 Issue 2 - 2015

\section{Gaida Albarqy, Mohammed Balghith* and} Moath Almallah

King Saud Bin Abdulaziz University for Health Sciences, Saudi Arabia

*Corresponding author: Mohammed Balghith, King Saud Bin Abdulaziz University for Health Sciences, Riyadh, P 0 Box 24490, Saudi Arabia, Tel: 00966504147204; Email: mbalghith@hotmail.com

Received: November 20, 2015 | Published: November 27, 2015
Abbreviations: CAD: Coronary Artery Disease; HDL: HighDensity Lipoprotein; BMI: Body Mass Index; CCU: Coronary Care Unit; STEMI: S-T Elevation Myocardial Infarction

\section{Introduction}

Coronary Artery Disease (CAD) is defined as obstruction of the lumen of the coronary artery due to formation of atherosclerotic plaque which contains cholesterol streaks. This eventually leads to reduced blood flow and blood supply to the myocardium. Patients having this problem will complain of chest pain on exertion due to reduction in the coronary perfusion which leads to a reduction in supply of oxygen to the myocardium. This results in an imbalance between oxygen supply and oxygen demand at myocardial cell level. In later stages, the obstruction is complete; there is a total obstruction of the vessel which later causes myocardial infarction. CAD is believed to initiate with injury to the internal layer of a coronary artery, at times it occurs as early as childhood [1].

CAD is a global problem, used to be the disease of developed countries, but recently it is becoming a disease of all countries. $\mathrm{CAD}$ is an overwhelming illness surely because a well individual in the beginning of his lifespan could decease or become disabled without notice. When the affected person is under 40 years of age, the catastrophic results for friends, family and jobs are particularly disastrous and sudden. The incidence of symptomatic CAD and heart attacks in young adults is low; majority of trials show $3 \%$ of all CAD cases happen within this age group [2]. The manifestation of CAD in younger patients is quite uncommon, unlike the general population. However, patients who seek medical attention due to symptomatic disease may signify the "tip of the iceberg" when bearing in mind manifest and subclinical disease all together. Young, asymptomatic patients do not usually undergo medical testing, this could explain the underestimated prevalence. When a repeated intracoronary ultrasound-related investigation was carried out in a series study of recent heart transplants (average donor age $33.4 \pm 13.2$ years) by Tuzcu et al. [3], the prevalence of CAD was more than half, with one in six teenagers showing coronary atherosclerosis.

Isolated risk factors were found in younger patients regarding CAD. Although noticeably high levels of cholesterol, LDL, and TG were seen in some young CAD patients, few studies have evaluated lipid profiles in those patients. Isser et al. [4] showed substantial increase in TGs and lipoprotein (a) (Lp[a]) levels and a decrease in high-density lipoprotein (HDL) cholesterol in young patients coming with a first episode of MI. They found a strong familial grouping of high level Lp(a) in the asymptomatic relatives of those patients. 
In a Finns study, high youth LDL-C and systolic HTN showed to be independent risk factors of middle age CAD, showing adolescence risk factor levels play a significant role in the formation of coronary atherosclerosis [5]. Usually insulindependent DM patients carry a high risk for CAD, the incidence of diabetes was seen in $15 \%$ to $20 \%$ of the young patients with CAD [6]. Recent data suggest high homocysteine and high Lp(a) are independent CAD predictors for young men. In an old study it was found that there was not a difference in the type of CAD when comparing young women to other patients. According to their coronary angiography which were done in 239 women less than 45 years of age [7].

In another study, an analysis of 35 patients out of 2400 patients with a mean age of 32 years showed smoking had been the most common risk factor in those young patients and other patients (89\%) [8]. The Saudi Study CADISS was one of the major studies in the kingdom and was conducted by King Saud University group showing the prevalence of CAD in urban and rural areas. The overall prevalence of CAD in KSA is 5.5\% in males and females were $6.6 \%$ and $4.4 \%$. Significant risk factors in KSA include: male gender, age, current smoking, fasting blood glucose, body mass index (BMI), hypertension, fasting cholesterol and triglycerides [9].

A study in Framingham, Massachusetts concluded that total cholesterol, blood pressure, and LDL cholesterol are predictors for CHD in the middle-aged white population [10]. In addition to the previous predictors, old age, male sex, low HDL-C, diabetes are associated with altered CHD events in patients with low LDL-C, according to MESA (Multi-Ethnic Study of Atherosclerosis) by the American College of Cardiology Foundation [11].

Studying the risk factors of CAD in younger Saudi patients of both sexes who underwent coronary angiography has not been shown yet. Which is why we are interested to identify in addition to classic risk factors, the rare and isolated risk factors of CAD in young adults aged 45 and below, according to their presentations to the hospital and to the morphology of their disease by angiogram. Identifying the important risk factors in our population would be important in order to improve the outcomes of our young generations.

\section{Methods}

\section{Aims}

The primary aim of this study is to identify the predictors of coronary disease on coronary angiography among young Saudi patients. The secondary aim is to study the prevalence of common risk factors in causing coronary artery disease in young Saudi patients.

\section{Setting}

The Study was conducted in the cardiac Cath. Lab in King Abdulaziz Cardiac Center at King Saud Bin Abdulaziz University for Health Sciences, Ministry of National Guard, Riyadh, Saudi Arabia.

KACC is one of the leading cardiac centers in the country. It was established by King Abdullah in April 2001. It contains adult and pediatric departments in both medical and surgical cardiac cases. There are more than 170 beds in the cardiac center. $60 \%$ of total admissions come through the emergency department. The cardiac center has three floors; a ground, first and second floor. The ground floor has out-patient clinics like general cardiac clinics for consultants, anticoagulation clinics, atherosclerosis clinics, and heart failure clinics, in addition to surgical and pediatric clinics. The ground floor also holds a cardiac rehabilitation center, a stress lab, and an echocardiography department. The first floor contains a Coronary Care Unit (CCU), a step-down unit and a cardiac surgical intensive unit for adult and pediatric. The second floor contains two cardiac wards and one surgical ward with almost equal number of beds.

The study is a retrospective, open label, non-randomized, single center study. Included patients are males and females from 18-45 years who underwent coronary angiography for various clinical indications in 2008- 2013 at King Abdul Aziz cardiac center. Exclusions are patients over the age of 45, patients under the age of 18 , patients with no coronary angiography, patients with congenital heart disease, patients with familial hyperlipidemia, and non-national guard eligible patients.

\section{Study protocol}

Patients between 18- 45 years $\rightarrow$ underwent coronary angiography from 2008-2013 at KACC. Patients were divided into two groups:

\section{Patients with obstructive CAD. \\ II. Patients with non-obstructive CAD.}

Baseline characteristics and baseline risk factors were obtained from the electronic chart review. Group comparisons were made to identify the independent predictors of CAD (both obstructive and non-obstructive) using logistic regression. Sample size were from 400-600 patients within 3 years.

a. Collection of All Data: We analyzed all coronary angiograms done in patients less than 45 years of age between the years of 2008-2013, a total of 36 months.

\section{Angiographic analysis}

a) Definition of Anatomy: Patients were divided into two groups; patients with abnormal and patients with normal coronary arteries. This analysis was done using the Xelera System by Philips Machines in the Catheterization laboratories at King AbdulAziz Cardiac Center with cardiologists to define the anatomy and abnormality of coronaries.

b) Defining Coronary Risk Factors: Baseline characteristics and baseline risk factors were obtained from the electronic chart review.

c) Group comparisons: were made to identify the independent predictors of CAD (both obstructive and non-obstructive)

All risk factors have been seen at in the chart particularly (Type 2 diabetes, hypertension, hyperlipidemia, smoking, and family history). 


\section{The statistical analysis}

Was planned using the Excel software. Continuous variables were analyzed using student $t$ test while categorical variables will be analyzed using Chi square test as appropriate. Multivariate logistic regression analysis were done to identify dependent and independent risk factors for abnormal coronaries. Both univariate and multivariate analysis will be made between the three groups comparing the prevalence of risk factors.

\section{Results}

A total of 1061patients who were admitted in King Abdul-Aziz Cardiac in National Guard Hospital from January 2008 to December 2013 with a clinical diagnosis of ischemic heart disease and underwent coronary angiography were studied according to their cardiac risk factors. By using Coronary angiography, obstructive coronary artery disease were found to be in 389 patients (37\%); 269 (25\%) were S-T Elevation myocardial Infarction (STEMI) and $120(11 \%)$ were Non-STEMI. All patients included in the study are patients aged 45 years and below. The majority of patients were male 889 (83\%), while females were 172 (17\%). The number of patients who gave a positive family history of coronary artery disease (CAD) were $104(10 \%)$. The number of patients diagnosed with hypertension and taking medication were 243 (23\%). Patients with hyperlipidemia were found to be 331 (29\%) most of whom were on anti-cholesterol medications. In addition to this finding, 305 (28\%) of total patients were defined to have obesity according to their BMI results. Type 2 Diabetes Mellitus were found in $227(26 \%)$ of the studied patients. Of the total number studied, 530 (50\%) were known smokers.

Risk factors for CAD have been classified into non-modifiable and modifiable. Modifiable risk factors include diabetes, obesity, hypertension, smoking, sedentary life style, and hypercholesterolemia. Non-modifiable include gender, age, and family history of CAD. The prevalence of cardiac risk factors studied in those patients are shown in the following Figures 1-4.

The results were then divided into two groups; Group A aged $<35$ and Group B >35years. Male gender in Gp.A 143(16.1\%) vs. Gp.B 746(83.9\%) p-value= 0.500. Smoking Gp.A $84(15.8 \%)$ vs. Gp.B 446(84.2\%) p=0.680. Obesity Gp.A 43(14.1\%) vs. Gp.B 262(85.9\%) p=0.234. Family history Gp.A 24(23.1\%) vs. $80(76.9 \%) \mathrm{p}=0.069$. Hyperlipidemia Gp.A $40(12.9 \%)$ vs. Gp.B 271(87.1\%) p=0.045. Hypertension Gp.A 20(8.2\%) vs. Gp 233(91.8\%) $\mathrm{p}=<0.001$. Diabetes Gp.A 20(7.2\%) vs.Gp.B $257(92.8 \%) \mathrm{p}=<0.001$.

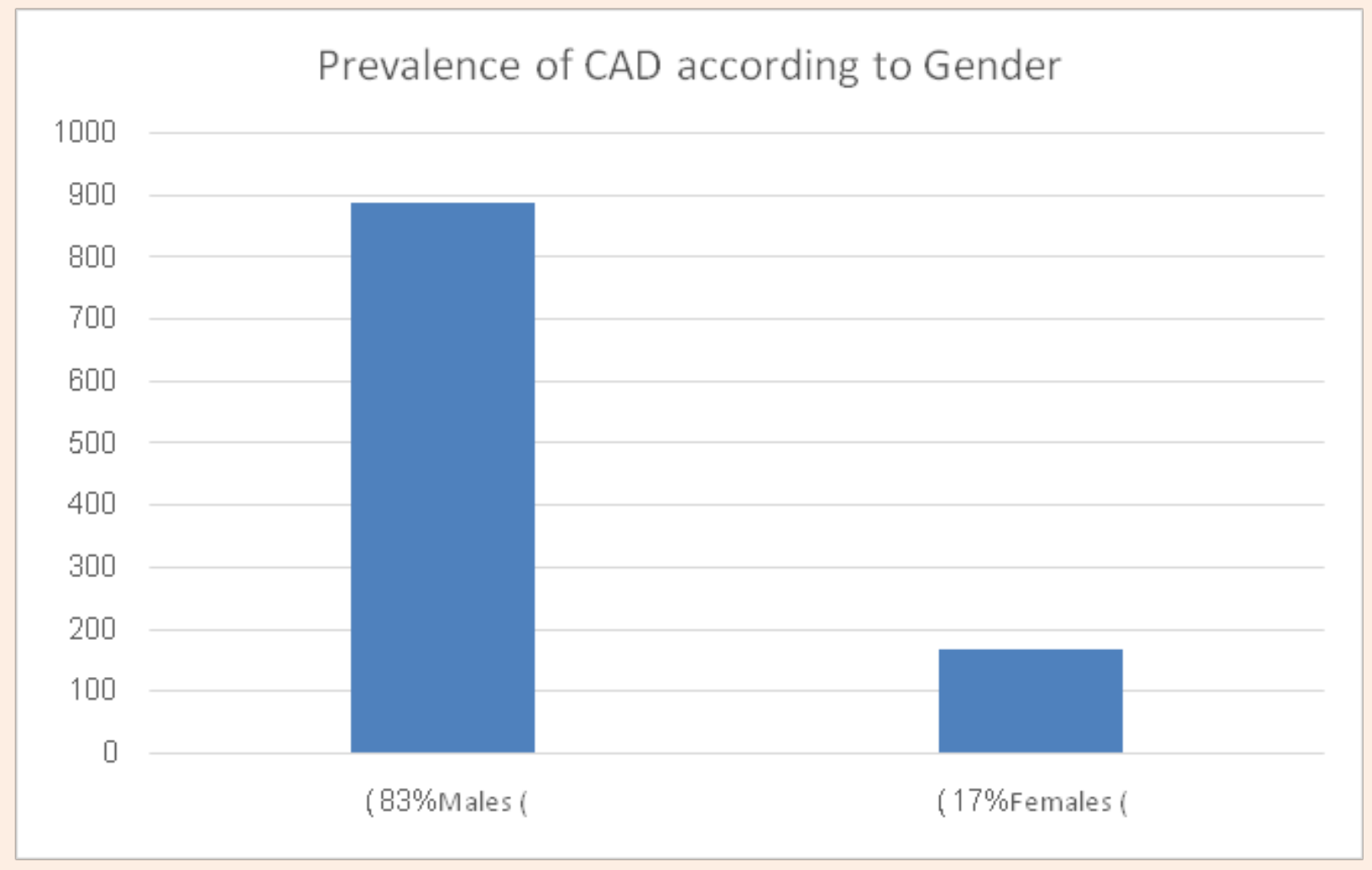

Figure 1: Showing prevalence of CAD among Saudi males and females. 


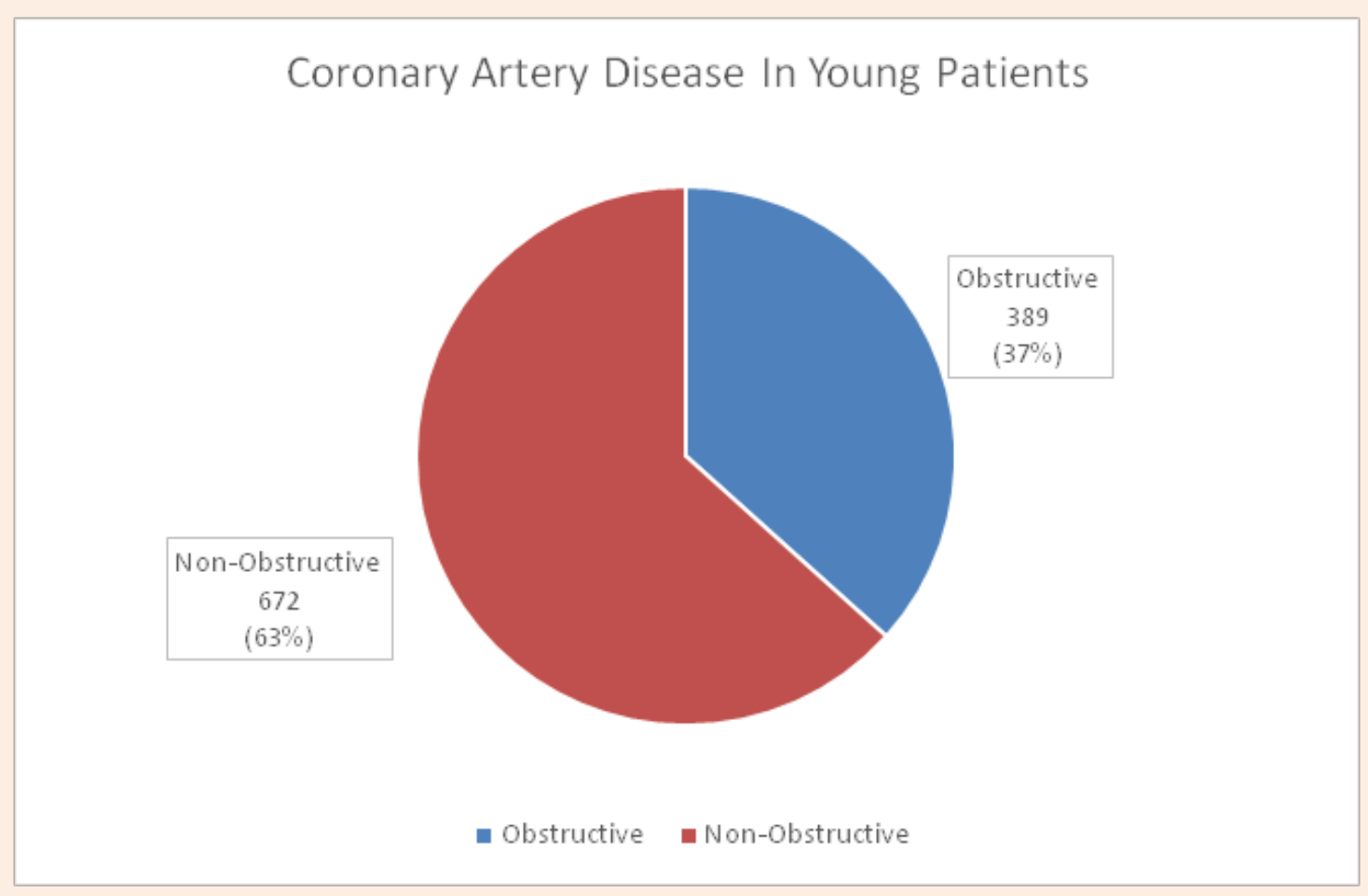

Figure 2: Showing the distribution of CAD in young patients.

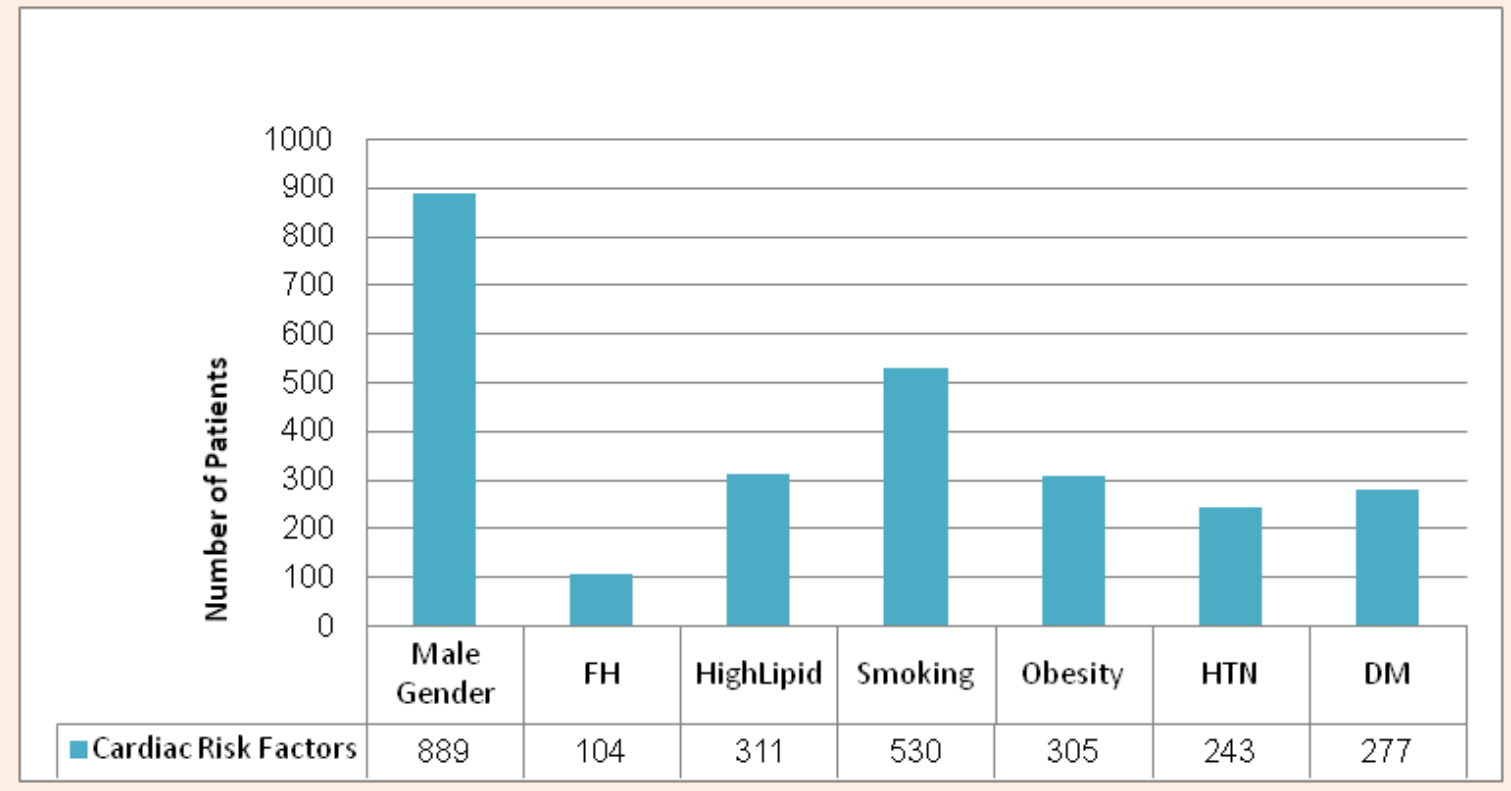

Figure 3: Showing the prevalence of Cardiac Risk Factors among Saudis below 45 years. 


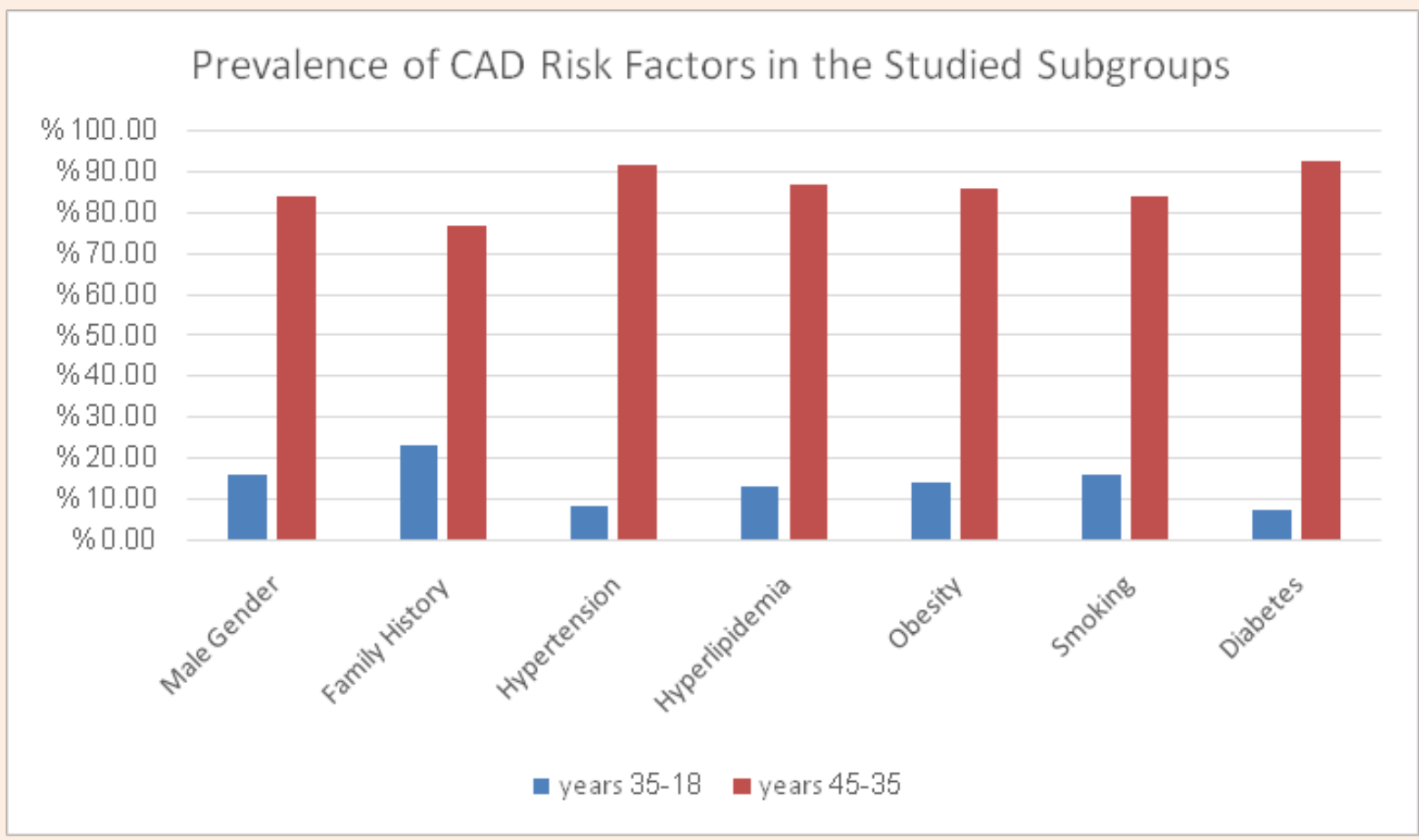

Figure 4: Showing the prevalence of CAD risk factors among the subgroups A ( $<35 y e a r s)$ and Group B ( $>45$ years).

\section{Discussion}

Risk factors for CAD have been classified into non-modifiable and modifiable. Modifiable risk factors include diabetes, obesity, hypertension, smoking, sedentary life style, and hypercholesterolemia. Non-modifiable include gender, age, and family history of CAD. These risk factors may occur alone or may intertwine leading to one another such as obesity leading to hypertension and diabetes. The more risk factors a patient has the higher the possibility of having CAD. A good common example worldwide would be Metabolic syndrome; a combination of hypertension, elevated insulin levels, high waist circumference, and high triglyceride levels, this can occur even in the younger age groups $<45$ years and highly increases with age. There is a high number of patients who live a sedentary lifestyle most of whom were found to have a high score of metabolic syndrome according to a study by Piotrowicz K et al. [12].

Our study was unique because we have focused on a younger Saudi group $<45$ years old. Our findings showed that the most significant risk factors were male gender (83\%) and smoking (50\%). Due to modern lifestyles and easy accessibility, smoking has become common among the younger population. This might explain the high incidence of smoking as major risk factors in this age group. In large study of CT-coronary angiogram to predict CAD either with obstructive stenosis $>50 \%$ or Plaque formation, those lesions were present in young men more than women, and according to this study, male sex was the strongest predictor for any CAD [13].
In a recent study conducted in Kuwait on patients younger than 35 years who underwent diagnostic cardiac catheterization concluded that smoking was the highest predictor of significant CAD in $70 \%$ of those young patients [14]. In a systematic review of 3885 studies done in India. It was found that the main risk factors contributing to CAD in all ages were in the following order: smoking, HTN and diabetes [15]. In a GRACE study there was a strong association between cigarette smoking and more STelevation MI at early presentation in younger patients with ACS [16]. CAD is one of the major reasons for sudden cardiac death in adults showed by a study from Montreal, Canada conducted on autopsies of patients aged above 20 years. This study also revealed that in young patients 30-40 years old, obesity and hyperlipidemia were the major predictors in this group of patients [17]. In a study made to assess the risk of CAD in patients who are obese but free of metabolic risk factors, it was found that although patients are only obese the progression of obesity may contribute to amplified risk for incidental CAD especially in young men [18].

A recent multicenter study from north india showed a significant increase in the lipid levels in younger patients $<45$ years with CAD vs older patients [19]. With the change of life style in Saudi Arabia and the introduction of western foods in addition to the traditional Saudi foods which are high in saturated fats we have found that hyperlipidemia was the third common risk factor among this study group. Most studies showed an important link between hyperlipidemia and obesity. Our study also supports the link between the two revealing the incidence of obesity to be $28 \%$ which we also believe is co-related. 
The prevalence of diabetes in the Saudi population was between $28-30 \%$ as published in studies previously. In our study the incidence of diabetes mellitus as a risk factor for coronary artery disease was found to be $26 \%$. In addition, prevalence of Hypertension was 23\%. A recent study done in Egypt in young metabolic syndrome patients, it was found that CAD and acute coronary syndrome weremore severe in this group of patients who are known cases of obesity, hyperlipidemia and DM [20]. Diabetes and hyperlipidemia are common among young CAD patients. These risk factors play big roles in the pathogenesis of CAD and their powerful influence to quicken the progression of atherosclerosis is well documented in the literature [21]. The lowest risk factor among the patients studied was having family history of CAD which accounted for $10 \%$ of the population studied. This indicates that in our society CAD in younger age less than 45 is not a familial disease and it is mainly related to male gender and life style habits such as smoking.

\section{Conclusion/Recommendations}

The prevalence of CAD was found the highest in male gender, smoking, and obesity respectively. In patients $>35$ years we found that HTN and Diabetes were statistically significant risk factors. Therefore, a modification of lifestyle habits is recommended.

\section{Study Limitation}

This study was a single center study, and patient numbers and samples are medium-sized compared with international centers.

\section{Acknowledgment}

Special thanks to catheterization laboratory staff including consultants, residents, nurses, technicians and to all who entered data into computers at KACC.

\section{References}

1. Mallika V, Goswami B, Rajappa M (2007) Atherosclerosis pathophysiology and the role of novel risk factors: a clinicobiochemical perspective. Angiology 58(5): 513-522.

2. Jalowiel DA, Hill JA (1989) Myocardial infarction in the young and in women. Cardiovasc Clin 20(1): 197-206.

3. Tuzcu EM, Kapadia SR, Tutar E, Ziada KM, Hobbs RE, et al. (2001) High prevalence of coronary atherosclerosis in asymptomatic teenagers and young adults: evidence from intravascular ultrasound. Circulation 103(22): 2705-2710.

4. Isser HS, Puri VK, Narain VS, Saran RK, Dwivedi SK, et al. (2001) Lipoprotein (a) and lipid levels in young patients with myocardial infarction and their first-degree relatives. Indian Heart J 53(4): 463466.

5. Hartiala O, Magnussen CG, Kajander S, Knuuti J, Ukkonen H, et al. (2012) Adolescence Risk Factors Are Predictive of Coronary Artery Calcification at Middle Age The Cardiovascular Risk in Young Finns Study. J Am Coll Cardiol 60(15): 1364-1370.

6. Foody JM, Milberg JA, Robinson K, Pearce GL, Jacobsen DW, et al. (2000) Homocysteine and lipoprotein (a) interact to increase CAD risk in young men and women. Arterioscler Thromb Vasc Biol 20(2): 493-499.
7. Waters DD, Halphen C, Theroux P, Paul-Robert D, Mizgala HF (1978) Coronary artery disease in young women: clinical and angiographic features and correlation with risk factors. Am J Cardiol J 42(1): 4147.

8. Wolfe MW, Vacek JL (1988) Myocardial infarction in the young. Angiographic features and risk factor analysis of patients with myocardial infarction at or before the age of 35 years. Chest 94(5): 926-930.

9. Al-Nozha MM, Arafah MR, Al-Mazrou YY, Al-Maatouq MA, Khan NB, et al. (2004) Coronary artery disease in Saudi Arabia. Saudi Med J 25(9): 1165-1171.

10. Wilson PW, D'Agostino RB, Levy D, Belanger AM, Silbershatz H, et al. (1998) Prediction of Coronary Heart Disease Using Risk Factor Categories. Circulation 97(18): 1837-1847.

11. Blankstein R, Budoff MJ, Shaw LJ, Goff DC Jr, Polak JF, et al. (2011) Predictors of Coronary Heart Disease Events Among Asymptomatic Persons With Low Low-Density Lipoprotein Cholesterol MESA (Multi-Ethnic Study of Atherosclerosis). J Am Coll Cardiol 58(4): 364-374.

12. Arzamendi $\mathrm{D}^{1}$, Benito B, Tizon-Marcos H, Flores J, Tanguay JF, et al. (2011) Increase in Sudden Death from Coronary Artery Disease in Young Adults, Am Heart J 161(3): 574-580.

13. Otaki Y, Gransar H, Cheng VY, Dey D, Labounty T, et al. (2015) Gender differences in the prevalence, severity, and composition of coronary artery disease in the young: a study of 1635 individuals undergoing coronary CT angiography from the prospective, multinational confirm registry. Eur Heart J Cardiovasc Imaging 16(5): 490-499.

14. Christus T, Shukkur AM, Rashdan I, Koshy T, Alanbaei M, et al. (2011) Coronary Artery Disease in Patients Aged 35 or less-A Different Beast? Heart Views 12(1): 7-11.

15. Rao M, Xavier D, Devi P, Sigamani A, Faruqui A, et al. (2015) Prevalence, treatments and outcomes of coronary artery disease in Indians: A systematic review. Indian Heart J 2015 67(4): 302-310.

16. Himbert D, Klutman M, Steg G, White K, Gulba DC, et al. (2005) Cigarette smoking and acute coronary syndromes: a multinational observational study . Int J Cardiol 100(1): 109-117.

17. Piotrowicz K, Pałkowska E, Bartnikowska E, Krzesiński P, Stańczyk A, et al. (2015) Self-reported health-related behaviors and dietary habits in patients with metabolic syndrome. Cardiol J 22(4): 413420 .

18. Twig G, Gerstein HC, Ben-Ami Shor D, Derazne E, Tzur D, et al (2015) Coronary artery disease risk among obese metabolically healthy young men. Eur J Endocrinol 173(3): 305-312.

19. Sinha N, Kumar S, Rai H, Singh N, Kapoor A, et al. (2012) Patterns and determinants of dyslipidaemia in 'Young' versus 'Not so Young' patients of coronary artery disease: a multicentric, randomised observational study in northern India. Indian Heart J 64(3): 229235.

20. Hassanin N, Gharib S, El Ramly MZ, Meged MA, Makram A (2015) Metabolic syndrome and coronary artery disease in young Egyptians presenting with acute coronary syndrome. Kasr Al Ainy Med J 21(1): 27-33.

21. Klein LW, Nathan S (2003) Coronary artery disease in young adults. J Am Coll Cardiol 41(4): 529-531. 